\title{
The Schroeder-Bernstein index for Banach spaces
}

\author{
by \\ Elói Medina Galego (São Paulo)
}

\begin{abstract}
In relation to some Banach spaces recently constructed by W. T. Gowers and B. Maurey, we introduce the notion of Schroeder-Bernstein index $\operatorname{SBi}(X)$ for every Banach space $X$. This index is related to complemented subspaces of $X$ which contain some complemented copy of $X$. Then we establish the existence of a Banach space $E$ which is not isomorphic to $E^{n}$ for every $n \in \mathbb{N}, n \geq 2$, but has a complemented subspace isomorphic to $E^{2}$. In particular, $\operatorname{SBi}(E)$ is uncountable. The construction of $E$ follows the approach given in 1996 by W. T. Gowers to obtain the first solution to the Schroeder-Bernstein Problem for Banach spaces.
\end{abstract}

1. Introduction. Let $X$ and $Y$ be Banach spaces. We write $X \stackrel{c}{\hookrightarrow} Y$ if $X$ is isomorphic to a complemented subspace of $Y, X \sim Y$ if $X$ is isomorphic to $Y$, and $X \nsim Y$ when $X$ is not isomorphic to $Y$. If $n \in \mathbb{N}=\{1,2, \ldots\}$, then $X^{n}$ denotes the sum of $n$ copies of $X$. The first infinite cardinal number will be denoted by $\aleph_{0}$ and the first uncountable cardinal by $\aleph_{1}$.

In 1996 W. T. Gowers [6] presented the first solution to the SchroederBernstein Problem for Banach spaces, that is, he constructed Banach spaces $X_{1}$ and $X_{2}$ such that

$$
X_{1} \stackrel{c}{\hookrightarrow} X_{2}, \quad X_{2} \stackrel{c}{\hookrightarrow} X_{1}, \quad X_{1} \not X_{2}
$$

Afterwards, in 1997, for each $p \in \mathbb{N}, p \geq 2$, W. T. Gowers and B. Maurey $\left[8\right.$, p. 563] defined a finite sequence of Banach spaces $X_{1}, \ldots, X_{p}$ such that for all $m, n \leq p$, we have

$$
X_{m} \stackrel{c}{\hookrightarrow} X_{n}, \quad X_{m} \nsim X_{n} \quad \text { for } m \neq n .
$$

Such sequences show that the structure of the complemented subspaces of a Banach space $X$ which contain some complemented copy of $X$ may be complicated. To examine this structure more closely it is natural to introduce the following definition:

2000 Mathematics Subject Classification: Primary 46B03, 46B20.

Key words and phrases: complemented subspaces, square of Banach space, SchroederBernstein property. 
Definition 1.1. Let $\kappa$ be a cardinal number, $\kappa \geq 2$. We say that a Banach space $X$ has the $\kappa$-Schroeder-Bernstein Property ( $\kappa$-SBP) if for each family $\left(X_{\xi}\right)_{\xi \in \kappa}$ of Banach spaces of cardinality $\kappa$ which satisfies

(a) $X \sim X_{1}$;

(b) $X_{\xi} \stackrel{c}{\hookrightarrow} X_{\gamma}, \forall \xi, \gamma \in \kappa$;

(c) $X_{\xi} \nsim X_{\gamma}, \forall \xi, \gamma \in \kappa$ with $\xi \neq 1, \gamma \neq 1$ and $\xi \neq \gamma$,

we have $X_{1} \sim X_{\xi}$ for some $\xi \in \kappa, \xi \neq 1$.

The Schroeder-Bernstein index $\operatorname{SBi}(X)$ of $X$ is defined by

$$
\operatorname{SBi}(X)=\inf \{\kappa: X \text { has the } \kappa-\operatorname{SBP}\} .
$$

Observe that this index is clearly well defined, and $\operatorname{SBi}(X)=2$ if and only if $X$ has the Schroeder-Bernstein Property (see [1]).

It follows directly from the definition that the Banach space $X_{1}$ in (1.1) satisfies $\operatorname{SBi}\left(X_{1}\right)>2$; see also [2]-[4] for more examples of such spaces. Furthermore, the space $X_{1}$ in (1.2) satisfies $\operatorname{SBi}\left(X_{1}\right)>p$.

The aim of this paper is to provide a Banach space $E$ with $\operatorname{SBi}(E)>\aleph_{0}$. In fact, we will show how to construct a Banach space $E$ with the properties announced in the abstract. Then Lemma 3.5 implies that $\operatorname{SBi}(E)$ is uncountable, because defining $X_{n}=E^{n}$ for $n \in \mathbb{N}$, we have

$$
X_{m} \stackrel{c}{\hookrightarrow} X_{n}, \quad X_{m} \nsim X_{n} \quad \text { for } m \neq n .
$$

The construction of $E$ is inspired by the papers [5] and [6]. In the first one, Gowers defined Banach spaces $X_{1}$ and $X_{2}$ such that $X_{1} \stackrel{c}{\hookrightarrow} X_{2}$ and $X_{1} \stackrel{c}{\hookrightarrow} X_{2}$, but $X_{1}^{m} \nsim X_{2}^{n}$, for all $m, n \in \mathbb{N}$ (see [3]). In the second, he exhibited the first Banach space $X$ satisfying $X \sim X^{3}$ and $X \nsim X^{2}$.

2. Preliminaries. As in [5] (see also [3]), we begin by fixing two totally incomparable Banach spaces $X$ and $Y$ from the class of sequence spaces constructed in [7]. We recall that the support of a vector $x=\left(x_{n}\right)_{n \in \mathbb{N}}$ in a sequence space, written $\operatorname{supp}(x)$, is $\left\{n \in \mathbb{N}: x_{n} \neq 0\right\}$. We will write $x<y$ to mean $i<j$ for every $i \in \operatorname{supp}(x)$ and $j \in \operatorname{supp}(y)$. If $x_{1}<\cdots<x_{m}$, we will say that the vectors $x_{1}, \ldots, x_{m}$ are successive.

We know that $X$ and $Y$ contain normalized sequences $x_{1}<x_{2}<\cdots$ and $y_{1}<y_{2}<\cdots$ respectively such that if $T: X \rightarrow X$ and $L: Y \rightarrow Y$ are any bounded linear operators then there exist $\lambda$ and $\mu$ such that $T\left(x_{n}\right)-\lambda x_{n} \rightarrow 0$ and $L\left(y_{n}\right)-\mu y_{n} \rightarrow 0$ as $n \rightarrow \infty$ (see [7, Lemma 22]). Moreover, we can prove in a way similar to the proof of [7, Lemma 23] that if $U: X \rightarrow Y$ and $V: Y \rightarrow X$ are any bounded linear operators, then $U\left(x_{n}\right) \rightarrow 0$ and $V\left(x_{n}\right) \rightarrow 0$ as $n \rightarrow \infty$.

Let $\left(x_{n}^{*}\right)_{n \in \mathbb{N}}$ and $\left(y_{n}^{*}\right)_{n \in \mathbb{N}}$ be sequences of support functionals for $\left(x_{n}\right)_{n \in \mathbb{N}}$ and $\left(y_{n}\right)_{n \in \mathbb{N}}$ respectively. 
We also fix two sequences $\left(X_{n}\right)_{n \in \mathbb{N}}$ and $\left(Y_{n}\right)_{n \in \mathbb{N}}$ of isometric copies of $X$ and $Y$ respectively. Let $Z_{n}=\left(X_{n} \oplus Y_{n}\right)_{\infty}$ be the sum of the Banach spaces $X_{n}$ and $Y_{n}$, with the supremum norm. We denote by $\bar{P}_{n}$ and $\bar{Q}_{n}$ the canonical projections of $Z_{n}$ onto $X_{n}$ and $Y_{n}$ respectively.

Let $V$ denote the vector space of all sequences $v=\left(z_{1}, z_{2}, z_{3}, \ldots\right)$ such that $z_{n} \in Z_{n}$ for all $n \in \mathbb{N}$ and $z_{n} \neq 0$ for only finitely many $n$. We identify each $Z_{n}$ with the subspace of $V$ given by $\left\{\left(0, \ldots, z_{n}, 0, \ldots\right): z_{n} \in Z_{n}\right\}$.

By $x_{i j}$ and $y_{i j}$, for $i, j \in \mathbb{N}$, we denote the elements in $V$ given respectively by

$$
z_{l}=\left\{\begin{array}{ll}
\left(x_{i}, 0\right) & \text { if } l=j, \\
0 & \text { if } l \neq j ;
\end{array} \quad z_{l}= \begin{cases}\left(0, y_{i}\right) & \text { if } l=j, \\
0 & \text { if } l \neq j .\end{cases}\right.
$$

The functionals $x_{i j}^{*}, y_{i j}^{*}$ and $w_{i j}^{*}$ are defined by

$$
\begin{aligned}
& x_{i j}^{*}(v)=x_{i}^{*}\left(\bar{P}_{j}\left(z_{j}\right)\right), \quad y_{i j}^{*}(v)=y_{i}^{*}\left(\bar{Q}_{j}\left(z_{j}\right)\right), \\
& w_{i j}^{*}(v)=x_{i}^{*}\left(\bar{P}_{j}\left(z_{j}\right)\right)-y_{i}^{*}\left(\bar{Q}_{j}\left(z_{j}\right)\right),
\end{aligned}
$$

for $v \in V, v=\left(z_{1}, z_{2}, z_{3}, \ldots\right), i, j \in \mathbb{N}$.

Let us fix three disjoint infinite subsets $\mathbb{N}_{1}, \mathbb{N}_{2}$ and $\mathbb{N}_{3}$ of $\mathbb{N}$. Now we introduce a convenient norm in $V$. If $v \in V, v=\left(z_{1}, z_{2}, z_{3}, \ldots\right)$, then $\|v\|$ is the maximum of the following three numbers:

$$
\left\{\begin{array}{l}
\sup \left\{\left\|z_{i}\right\|: i \in \mathbb{N}\right\} ; \\
\sup \left\{\left(\sum_{j=1}^{\infty}\left(\left|x_{i j}^{*}(v)\right|^{4}+\left|y_{i j}^{*}(v)\right|^{4}\right)\right)^{1 / 4}: i \in \mathbb{N}_{2}\right\} \\
\sup \left\{\left(\sum_{j=1}^{\infty}\left|w_{i j}^{*}(v)\right|^{2}\right)^{1 / 2}: i \in \mathbb{N}_{3}\right\} .
\end{array}\right.
$$

Let $Z$ be the completion of $(V,\|\ldots\|)$.

Note that $Z_{n}$ is closed in $Z$, because $\left\|z_{n}\right\| \leq\left\|\left(0, \ldots, z_{n}, 0, \ldots\right)\right\| \leq$ $2^{1 / 2}\left\|z_{n}\right\|$ for all $z_{n} \in Z_{n}$. Moreover, $Z$ is equal to the closed subspace generated by $\left(Z_{n}\right)_{n \in \mathbb{N}}$ in $Z$, and $\left\|\sum_{i=1}^{n} z_{i}\right\| \leq\left\|\sum_{i=1}^{n+m} z_{i}\right\|$ for every $z_{i} \in Z_{i}$ and $m, n \in \mathbb{N}$. Therefore [10, Theorem 15.5] implies that $\left(Z_{n}\right)_{n \in \mathbb{N}}$ is a Schauder decomposition of $Z$. We will write

$$
Z=Z_{1} \oplus Z_{2} \oplus Z_{3} \oplus \cdots
$$

It is easy to see that this sum is symmetric, that is, the norm of an element $\left(z_{n}\right)_{n \in \mathbb{N}}$ of this sum is not affected by changing the order of the terms or by multiplying some of them by -1 . Thus

$$
Z \sim Z_{1} \oplus Z_{3} \oplus Z_{5} \oplus \cdots, \quad Z \sim Z_{2} \oplus Z_{4} \oplus Z_{6} \oplus \cdots
$$

and

$$
Z \sim\left(Z_{1} \oplus Z_{3} \oplus Z_{5} \oplus \cdots\right) \oplus\left(Z_{2} \oplus Z_{4} \oplus Z_{6} \oplus \cdots\right)
$$


Consequently,

$$
Z \sim Z^{2}
$$

For every $n \in \mathbb{N}$, we define

$$
W_{n}=Z_{n+1} \oplus Z_{n+2} \oplus Z_{n+3} \oplus \cdots .
$$

Let $S$ denote the shift operator defined by $S(v)=\left(0, z_{1}, z_{2}, \ldots\right)$ for $v \in Z$, $v=\left(z_{1}, z_{2}, z_{3}, \ldots\right)$. It is clear from the definition of $Z$ that $S^{n}$ is an isometry from $Z$ onto $W_{n}$ for every $n \in \mathbb{N}$. So, for every $n \in \mathbb{N}$, we have

$$
Z \sim W_{n} .
$$

Now we put $E=X \oplus Z$. Hence $E$ contains a complemented subspace isomorphic to its square $E^{2}$. Indeed, by (2.1) and (2.2) we get

$$
E^{2} \sim X^{2} \oplus Z^{2} \sim X^{2} \oplus W_{2} \sim X_{1} \oplus X_{2} \oplus W_{2} \stackrel{c}{\hookrightarrow} Z \stackrel{c}{\hookrightarrow} E .
$$

To obtain more information about the finite sums of $E, E^{p}$, for every $p \in \mathbb{N}$, $p \geq 2$, it will be useful to define the Banach spaces

$$
G_{p}=X_{1} \oplus X_{2} \oplus \cdots \oplus X_{p-1} \oplus W_{p-1} .
$$

We observe that $E=X \oplus Z \sim X_{1} \oplus W_{1}=G_{2}$ and again by (2.1) and (2.2),

$$
E^{p} \sim X^{p} \oplus Z^{p} \sim X^{p} \oplus W_{p} \sim G_{p+1} .
$$

Hence to prove that $E \not E^{p}$ for every $p \in \mathbb{N}, p \geq 2$, it suffices to show that

$$
G_{2} \nsim G_{p+1} \text {. }
$$

We will verify (2.3) by proving that $Y \oplus G_{2} \nsim Y \oplus G_{p+1}$, that is,

$$
Z \not G_{p}
$$

for every $p \in \mathbb{N}, p \geq 2$ (see Theorem 3.4).

For this purpose we need some more definitions. For any $j \in \mathbb{N}$ we let $P_{j}$ and $Q_{j}$ be the canonical projections of $Z$ onto $X_{j}$ and $Y_{j}$ respectively.

Let $T$ be a bounded linear operator from $Z$ to $G_{p}$. For any $i, j \in \mathbb{N}$ let $T_{i j}: X_{i} \rightarrow X_{j}$ be the restriction of $P_{j} T$ to $X_{i}$. Similarly, let $U_{i j}: X_{i} \rightarrow Y_{j}$, $L_{i j}: Y_{i} \rightarrow Y_{j}$ and $V_{i j}: Y_{i} \rightarrow X_{j}$ be the restrictions of $Q_{j} T$ to $X_{i}, Q_{j} T$ to $Y_{i}$ and $P_{j} T$ to $Y_{i}$ respectively.

As we have already said, we can associate scalars $\lambda_{j i}$ with each $T_{i j}$ and $\mu_{j i}$ with each $L_{i j}$, so that

$$
\begin{aligned}
T_{i j}\left(x_{n i}\right)-\lambda_{j i} x_{n j} & \rightarrow 0, & L_{i j}\left(y_{n i}\right)-\mu_{j i} y_{n j} & \rightarrow 0, \\
U_{i j}\left(x_{n i}\right) & \rightarrow 0, & V_{i j}\left(y_{n i}\right) & \rightarrow 0,
\end{aligned}
$$

as $n \rightarrow \infty$, for all $i, j \in \mathbb{N}$. In particular, we have defined two infinite matrices

$$
\Lambda=\left(\lambda_{i j}\right)_{i, j=1}^{\infty} \text { and } M=\left(\mu_{i j}\right)_{i, j=1}^{\infty} .
$$


$\mathcal{S}$ stands for the space of all sequences of scalars. Let $a=\left(a_{n}\right)_{n \in \mathbb{N}}$ in $\mathcal{S}$, $x \in X$ and $y \in Y$. We define

$$
a \ominus x=\left(a_{1} x, 0, a_{2} x, 0, a_{3} x, 0, \ldots\right), \quad a \ominus y=\left(0, a_{1} y, 0, a_{2} y, 0, a_{3} y, 0, \ldots\right) .
$$

Let $c_{00}$ be the space of sequences of scalars all but finitely many of which are zero. Set $\Lambda a=\left(\sum_{i=1}^{\infty} \lambda_{n i} a_{i}\right)_{n \in \mathbb{N}}$ for $a=\left(a_{n}\right)_{n \in \mathbb{N}}$ in $c_{00}$.

Denote by $\mathcal{Z}$ the vector space of sequences $u=\left(z_{1}, z_{2}, z_{3}, \ldots\right)$ such that $z_{j} \in Z_{j}$ for all $j \in \mathbb{N}$, and define $\mathcal{P}_{j}\left(z_{1}, z_{2}, z_{3}, \ldots\right)=\bar{P}_{j}\left(z_{j}\right)$ and $\mathcal{Q}_{j}\left(z_{1}, z_{2}, z_{3}, \ldots\right)=\bar{Q}_{j}\left(z_{j}\right)$, for $\left(z_{1}, z_{2}, z_{3}, \ldots\right) \in \mathcal{Z}$ and $j \in \mathbb{N}$.

Finally, we will consider $Z$ as a vector subspace of $\mathcal{Z}$. In particular, $P_{j}(u)=\mathcal{P}_{j}(u)$ and $Q_{j}(u)=\mathcal{Q}_{j}(u)$ for all $u \in Z$ and $j \in \mathbb{N}$.

3. The results. We are now prepared to obtain results similar to those in [5] for the spaces $Z$ and $G_{p}$.

Lemma 3.1. Let $T$ be a bounded linear operator from $Z$ to $G_{p}, p \geq 2$, and $a \in c_{00}$. Then for all $j \in \mathbb{N}$, as $n \rightarrow \infty$,

(a) $P_{j}\left(T\left(a \ominus x_{n}\right)\right)-\mathcal{P}_{j}\left(\Lambda a \ominus x_{n}\right) \rightarrow 0$.

(b) $Q_{j}\left(T\left(a \ominus x_{n}\right)\right) \rightarrow 0$.

(c) $Q_{j}\left(T\left(a \ominus y_{n}\right)\right)-\mathcal{Q}_{j}\left(\Lambda a \ominus y_{n}\right) \rightarrow 0$.

(d) $P_{j}\left(T\left(a \ominus x_{n}\right)\right) \rightarrow 0$.

Proof. By symmetry it suffices to prove (a) and (b).

(a) We have

$$
P_{j}\left(T\left(a \ominus x_{n}\right)\right)=P_{j}\left(\sum_{i=1}^{\infty} a_{i} T\left(x_{n i}\right)\right)=\sum_{i=1}^{\infty} a_{i} T_{i j}\left(x_{n i}\right) .
$$

So by the definition of $\lambda_{j i}$,

$$
P_{j}\left(T\left(a \ominus x_{n}\right)\right)-\left(\sum_{i=1}^{\infty} a_{i} \lambda_{j i}\right) x_{n j} \rightarrow 0 \quad \text { as } n \rightarrow \infty .
$$

But the second term in the above expression is $\mathcal{P}_{j}\left(\Lambda a \ominus x_{n}\right)$.

(b) It suffices to note that $Q_{j}\left(T\left(a \ominus x_{n}\right)\right)=\sum_{i=1}^{\infty} a_{i} U_{i j}\left(x_{n i}\right)$.

Lemma 3.2. If $\left(v_{i}\right)_{i=1}^{q}$ are successive elements of $Z$, then

$$
\left\|\sum_{i=1}^{q} v_{i}\right\| \leq\left(\sum_{i=1}^{q}\left\|v_{i}\right\|^{2}\right)^{1 / 2} .
$$

Proof. This follows from the definition of the norm in $Z$.

In order to prove the next lemma, we recall that $c_{0}, \ell_{\infty}, \ell_{2}$ and $\ell_{4}$ denote the classical Banach sequence spaces, and $\left\{e_{n}: n \in \mathbb{N}\right\}$ are their unit vectors. 
Lemma 3.3. If $T$ is an isomorphism from $Z$ onto $G_{p}, p \geq 2$, then $\Lambda$ is the matrix of an isomorphism from $c_{0}$ onto $c_{0}$, while $M$ is the matrix of an isomorphism between $c_{0}$ and its subspace $c_{0}^{(p-1)}$ generated by $\left\{e_{n}: n \geq p\right\}$.

Proof. Let $C \in \mathbb{R}$ be such that $\max \left\{\|T\|,\left\|T^{-1}\right\|\right\} \leq C$. First we show that $\Lambda$ represents a bounded linear operator from $c_{0}$ to $\ell_{\infty}$. If this were not the case, then there would exist $a \in c_{00}$ such that $\|a\|_{\infty}=1$ and $\|\Lambda a\|_{\infty} \geq$ $4 C$. Set $b=\Lambda a$ and let $j \in \mathbb{N}$ be such that $\left|b_{j}\right| \geq 3 C$. By Lemma 3.1, $P_{j}\left(T\left(a \ominus x_{n}\right)\right)-\mathcal{P}_{j}\left(b \ominus x_{n}\right) \rightarrow 0$ as $n \rightarrow \infty$. Since $\mathcal{P}_{j}\left(b \ominus x_{n}\right)=b_{j} x_{n}$ we can pick $n \in \mathbb{N}_{1}$ such that $\left\|P_{j} T\left(a \ominus x_{n}\right)\right\| \geq 2 C$, therefore $\left\|T\left(a \ominus x_{n}\right)\right\| \geq 2 C$. However, since $n \in \mathbb{N}_{1}$, we have $\left\|a \ominus x_{n}\right\|=\sup \left\{\left\|a_{i} x_{n}\right\|: i \in \mathbb{N}\right\}=\|a\|_{\infty}=1$, which is a contradiction.

A similar argument shows that $\Lambda$ is the matrix of a bounded linear operator from $\ell_{4}$ to $\ell_{4}$. Indeed, otherwise we could find $a \in c_{00}$ such that $\|a\|_{4}=1,\|b\|_{4} \geq 4 C$, where $b=\Lambda a$. Again, Lemma 3.1 implies that $P_{j}\left(T\left(a \ominus x_{n}\right)\right)-\mathcal{P}_{j}\left(b \ominus x_{n}\right) \rightarrow 0$ as $n \rightarrow \infty$. Choose $q \in \mathbb{N}$ such that $\left(\sum_{l=1}^{q}\left|b_{l}\right|^{4}\right)^{1 / 4} \geq 3 C$.

Now, by Lemma 3.1, we can choose $n \in \mathbb{N}_{2}$ such that

$$
\left|x_{n}^{*}\left(P_{j}\left(T\left(a \ominus x_{n}\right)\right)-b_{j} x_{n}\right)\right| \leq C / 2^{j}, \quad \forall j \leq q .
$$

By the definition of the norm in $G_{p}$ we have

$$
\left\|T\left(a \ominus x_{n}\right)\right\| \geq\left(\sum_{j=1}^{q}\left|x_{n j}^{*}\left(T\left(a \ominus x_{n}\right)\right)\right|^{4}\right)^{1 / 4} \geq\left(\sum_{j=1}^{q}\left|b_{j}\right|^{4}\right)^{1 / 4}-C \geq 2 C .
$$

Since $n \in \mathbb{N}_{2}$, we have $\left\|a \ominus x_{n}\right\| \leq\|a\|_{4}=1$, again giving a contradicition.

This shows in particular that the image of any element in $c_{0}$ under $\Lambda$ is in $c_{0}$. Hence $\Lambda$ represents a bounded linear operator from $c_{0}$ to $c_{0}$.

Note that the preceding arguments also show that $\Lambda\left(T^{-1}\right)$ (the matrix obtained from $T^{-1}$ ) represents a bounded linear operator from $c_{0}$ to $c_{0}$.

We will complete the proof by showing that both $\Lambda\left(T^{-1}\right) \Lambda(T)$ and $\Lambda(T) \Lambda\left(T^{-1}\right)$ are the identity on $c_{0}$.

Given any $n \in \mathbb{N}_{1}$ and $a \in c_{0},\|a\|=1$, it follows from the definition of the norm in $Z$ that $\Lambda a \ominus x_{n} \in G_{p}$. So, by Lemma 3.1 and the continuity of $T$ and $\Lambda$, we can write

$$
T\left(a \ominus x_{n}\right)=\Lambda a \ominus x_{n}+v_{n}
$$

with

$$
v_{n} \in G_{p}, \quad P_{j}\left(v_{n}\right) \rightarrow 0 \quad \text { as } n \rightarrow \infty, \forall j \in \mathbb{N} .
$$

Setting $\Lambda^{\prime}=\Lambda\left(T^{-1}\right)$, again by the analogue of Lemma 3.1 for $T^{-1}$, we can write

$$
T^{-1}\left(\Lambda a \ominus x_{n}\right)=\Lambda^{\prime}(\Lambda a) \ominus x_{n}+u_{n}
$$


with

$$
u_{n} \in Z, \quad P_{j}\left(u_{n}\right) \rightarrow 0 \quad \text { as } n \rightarrow \infty, \forall j \in \mathbb{N} .
$$

Therefore, by using (3.1) and (3.3), we deduce that

$$
a \ominus x_{n}=\Lambda^{\prime}(\Lambda a) \ominus x_{n}+u_{n}+T^{-1}\left(v_{n}\right) .
$$

The bounds of $\|\Lambda\|_{\infty},\left\|\Lambda^{\prime}\right\|_{\infty}$ and $\|T\|$ yield $\left\|v_{n}\right\| \leq 5 C$ and $\left\|u_{n}\right\| \leq 30 C^{2}$.

Suppose that there exists $a \in c_{0}$ with $\|a\|_{\infty}=1$ such that $\Lambda^{\prime}(\Lambda a)=c \neq a$, so $(a-c) \ominus x_{n}=u_{n}+T^{-1}\left(v_{n}\right)$. By using (3.2) and (3.4) we can find an infinite subset $\mathcal{N}$ of $\mathbb{N}_{1}$, a sequence $\left(u_{n}^{\prime}\right)_{n \in \mathbb{N}}$ of successive elements in $Z$ and a sequence $\left(v_{n}^{\prime}\right)_{n \in \mathbb{N}}$ of successive elements in $G_{p}$ such that $\left\|u_{n}^{\prime}-u_{n}\right\| \leq 100^{-n}$ and $\left\|v_{n}^{\prime}-v_{n}\right\| \leq 100^{-n}$ for all $n \in \mathcal{N}$.

Now, we pick $n_{1}, \ldots, n_{t}$ from $\mathcal{N}$. Then by $(3.5)$,

$$
\sum_{i=1}^{t}(a-c) \ominus x_{n_{i}}=\sum_{i=1}^{t} u_{n_{i}}^{\prime}+T^{-1}\left(\sum_{i=1}^{t} v_{n_{i}}^{\prime}\right)+u(t)
$$

where

$$
u(t) \in G_{p}, \quad\|u(t)\| \leq(1+C) \sum_{i=1}^{t} 100^{-n_{i}} .
$$

But for a suitable $j \in \mathbb{N}$ we have

$$
\left\|\sum_{i=1}^{t}(a-c) \ominus x_{n_{i}}\right\|=\|(a-c)\|_{\infty}\left\|\sum_{i=1}^{t} x_{n_{i} j}\right\| .
$$

However, since the vectors $x_{n_{1}}, \ldots, x_{n_{t}}$ are successive and normalized in $X$, it follows from the definition of the norm in $X[7, \mathrm{p} .863]$ that

$$
\left\|\sum_{i=1}^{t} x_{n_{i}}\right\| \geq \frac{t}{\log _{2}(t+1)} .
$$

On the other hand, by Lemma 3.2,

$$
\left\|\sum_{i=1}^{t} u_{n_{i}}^{\prime}\right\| \leq\left(\sum_{i=1}^{t}\left\|u_{n_{i}}^{\prime}\right\|^{2}\right)^{1 / 2} \leq 30 t^{1 / 2} C^{2}+\left(\sum_{i=1}^{t} 100^{-2 n_{i}}\right)^{1 / 2} \text {. }
$$

We also have, by Lemma 3.2,

$$
\begin{aligned}
\left\|T^{-1}\left(\sum_{i=1}^{t} v_{n_{i}}^{\prime}\right)\right\| & \leq C\left(\sum_{i=1}^{t}\left\|v_{n_{i}}^{\prime}\right\|^{2}\right)^{1 / 2} \\
& \leq 5 t^{1 / 2} C^{2}+C\left(\sum_{i=1}^{t} 100^{-2 n_{i}}\right)^{1 / 2} .
\end{aligned}
$$

Hence, by (3.7), (3.10) and (3.11), the right hand side of (3.6) has norm at most $35 t^{1 / 2} C^{2}+(1+C)\left(\sum_{i=1}^{t} 100^{-n_{i}}+\left(\sum_{i=1}^{t} 100^{-2 n_{i}}\right)^{1 / 2}\right)$. This contradicts (3.9) and (3.6) when $t$ is large enough. 
A similar proof shows that $\Lambda \Lambda^{\prime}$ is the identity on $c_{0}$, and finally a similar argument to the whole of this proof shows that $M$ is an isomorphism from $c_{0}$ onto $c_{0}^{(p-1)}$.

TheOREM 3.4. $Z$ is isomorphic to $G_{p}$ for no $p \in \mathbb{N}, p \geq 2$.

Proof. Suppose that $T$ is an isomorphism from $Z$ onto $G_{p}$. Then Lemma 3.3 implies that $\Lambda: c_{0} \rightarrow c_{0}$ is Fredholm with index 0 , and $M$ is Fredholm with index $p-1$. We know that $\Lambda-M$ cannot be strictly singular (see [9, Proposition 2.c.10]). In particular, we can find $\delta>0$ and a sequence $a_{1}<a_{2}<\cdots$ of elements in $c_{00}$ of norm one such that $\left\|(\Lambda-M)\left(a_{n}\right)\right\| \geq \delta$ for every $n \in \mathbb{N}$.

Let $C \in \mathbb{R}$ be such that $\max \left\{\|T\|,\left\|T^{-1}\right\|\right\} \leq C$ and choose $q>(4 C / \delta)^{2}$, $q \in \mathbb{N}$.

Since $\left(\Lambda a_{n}\right)_{i} \rightarrow 0$ and $\left(M a_{n}\right)_{i} \rightarrow 0$ as $n \rightarrow \infty$ for all $i \in \mathbb{N}$, we can obtain integers $0=n_{0}<n_{1}<\cdots<n_{q}$ and $q$ elements of $\left\{a_{n}: n \in \mathbb{N}\right\}$ such that after relabelling these elements as $a_{1}, \ldots, a_{q}$, we have

$$
\sup _{i \notin\left[n_{l-1}+1, n_{l}\right]}\left|\left(\Lambda\left(a_{l}\right)\right)_{i}\right|<\frac{\delta}{8 q}, \quad l=1, \ldots, q,
$$

and

$$
\sup _{i \notin\left[n_{l-1}+1, n_{l}\right]}\left|\left(M\left(a_{l}\right)\right)_{i}\right|<\frac{\delta}{8 q}, \quad l=1, \ldots, q .
$$

Let $j_{l}$ with $n_{l-1}+1 \leq j_{l}<n_{l}$ be such that

$$
\left|\left((\Lambda-M)\left(a_{l}\right)\right)_{j_{l}}\right| \geq \delta, \quad l=1, \ldots, q .
$$

According to Lemma 3.1, we know that

$$
\left\|T\left(\sum_{n=1}^{q}\left(a_{n} \ominus x_{k}+a_{n} \ominus y_{k}\right)\right)\right\|=\left\|\sum_{n=1}^{q}\left(\Lambda a_{n} \ominus x_{k}+M a_{n} \ominus y_{k}\right)+u_{k}\right\|,
$$

where

$$
u_{k} \in \mathcal{Z}, \quad \mathcal{P}_{j}\left(u_{k}\right) \rightarrow 0, \quad \mathcal{Q}_{j}\left(u_{k}\right) \rightarrow 0 \quad \text { as } k \rightarrow \infty, \forall j \in \mathbb{N} .
$$

Fix $m$ with $1 \leq m \leq q$ and $k \in \mathbb{N}$ sufficiently large such that $\left\|\mathcal{P}_{j_{m}}\left(u_{k}\right)\right\|<$ $\delta / 8$ and $\left\|\mathcal{Q}_{j_{m}}\left(u_{k}\right)\right\|<\delta / 8$. Then

$$
\begin{aligned}
\mid w_{k j_{m}}^{*} & \left(\sum_{n=1}^{q}\left(\Lambda a_{n} \ominus x_{k}+M a_{n} \ominus y_{k}\right)+u_{k}\right) \mid \\
= & \mid\left((\Lambda-M)\left(a_{1}\right)\right)_{j_{m}}+\cdots+\left((\Lambda-M)\left(a_{m}\right)\right)_{j_{m}}+\cdots+\left((\Lambda-M)\left(a_{q}\right)\right)_{j_{m}} \\
& +x_{k}^{*}\left(\mathcal{P}_{j_{m}}\left(u_{k}\right)\right)-y_{k}^{*}\left(\mathcal{Q}_{j_{m}}\left(u_{k}\right)\right) \mid \\
\geq & \delta-2(q-1) \frac{\delta}{8 q}-\frac{\delta}{4} \geq \frac{\delta}{2} .
\end{aligned}
$$


So, by the definition of the norm in $Z$, for sufficiently large $k \in \mathbb{N}_{3}$ we obtain

$$
\left\|\sum_{n=1}^{q}\left(\Lambda a_{n} \ominus x_{k}+M a_{n} \ominus y_{k}\right)+u_{k}\right\| \geq \frac{\delta}{2} \sqrt{q} .
$$

On the other hand the action of each $w_{k j}^{*}$ is zero on every vector of the form $a \ominus x_{k}+a \ominus y_{k}$ with $a \in c_{00}$. Thus, again by the definition of the norm in $Z$ we have $\left\|\sum_{n=1}^{q}\left(a_{n} \ominus x_{k}+a_{n} \ominus y_{k}\right)\right\|=1$. Therefore by (3.15) and (3.17) we conclude that $\|T\| \geq \delta \sqrt{q} / 2$. Since we have chosen $q$ to be greater than $(4 C / \delta)^{2}$, we have shown that $\|T\|>C$. This contradiction completes the proof.

Lemma 3.5. Let $X$ be a Banach space such that $X^{2} \stackrel{c}{\hookrightarrow} X$. If $X^{p} \sim X^{q}$ for some $p, q \in \mathbb{N}$ with $p<q$, then $X \sim X^{q-p+1}$.

Proof. Since $X^{2} \stackrel{c}{\hookrightarrow} X$, it follows that $X^{p} \stackrel{c}{\hookrightarrow} X$. Therefore there exists a Banach space $B$ such that $X \sim X^{p} \oplus B$. Thus

$$
X^{q-p+1} \sim X^{q-p} \oplus X \sim X^{q-p} \oplus X^{p} \oplus B \sim X^{q} \oplus B \sim X^{p} \oplus B \sim X .
$$

4. Remarks and problems. The existence of the Banach space $E$ constructed in this paper leads in the natural way to some problems related to the structure of complemented subspaces in Banach spaces. We now mention some of them.

Problem 4.1. Does there exist a Banach space $X$ with $\operatorname{SBi}(X)=\aleph_{1}$ ?

Let $X$ be a Banach space with an unconditional basis. An unsolved problem is whether $\operatorname{SBi}(X)=2$ (see [8]). In fact, we do not even know the answer to

Problem 4.2. Let $X$ be a Banach space with an unconditional basis. Is it true that $\operatorname{SBi}(X)<\aleph_{0}$ ?

Now we state some natural problems concerning the Schroeder-Bernstein index. In [2] a Banach space $X$ with $2=\operatorname{SBi}\left(X^{*}\right)<\operatorname{SBi}(X)$ was exhibited, where $X^{*}$ is the dual space of $X$. Nevertheless Problems 4.3 and 4.4 below are still open even in the case where $\operatorname{SBi}(X)=2$ and $\operatorname{SBi}(Y)=2$ (see [1]).

Problem 4.3. Is it true that $\operatorname{SBi}\left(X^{*}\right) \leq \operatorname{SBi}(X)$ for every Banach space $X$ ?

Problem 4.4. Is it true that $\operatorname{SBi}(X \oplus Y) \leq \sup \{\operatorname{SBi}(X), \operatorname{SBi}(Y)\}$ for any Banach spaces $X$ and $Y$ ?

Finally, let $X$ represent the complex Banach space constructed by W. T. Gowers and B. Maurey in [8, Section 4.3]. Then $X$ is isomorphic to its subspaces of even codimension while not being isomorphic to those of odd codimension [8, Theorem 19]. In particular, $X \oplus \mathbb{C} \stackrel{c}{\hookrightarrow} X$ and $X$ 
is not isomorphic to $X \oplus \mathbb{C}$. Hence $\operatorname{SBi}(X) \geq 3$. Furthermore, if $Y$ is any infinite-dimensional complemented subspace of $X$, then either $Y \sim X$ or $Y \sim X \oplus \mathbb{C}$ (see remarks after [8, Theorem 19]), thus $\operatorname{SBi}(X)=3$.

Acknowledgments. We are grateful to V. Ferenczi for suggesting the search of the Banach space constructed in this work, and also to the referee for his comments on the previous version of the paper.

\section{References}

[1] P. G. Casazza, The Schroeder-Bernstein property for Banach spaces, in: Contemp. Math. 85, Amer. Math. Soc., 1989, 61-77.

[2] E. M. Galego, How to generate new Banach spaces non-isomorphic to their cartesian squares, Bull. Polish Acad. Sci. Math. 47 (1999), 21-25.

[3] -, Banach spaces complemented in each other without isomorphic finite sums, ibid. 50 (2002), 1-9.

[4] -, On solutions to the Schroeder-Bernstein problem for Banach spaces, Arch. Math. (Basel) 74 (2002), 299-307.

[5] W. T. Gowers, A solution to the Schroeder-Bernstein problem for Banach spaces, preliminary version (unpublished).

[6] - A solution to the Schroeder-Bernstein problem for Banach spaces, Bull. London Math. Soc. 28 (1996), 297-304.

[7] W. T. Gowers and B. Maurey, The unconditional basic sequence problem, J. Amer. Math. Soc. 6 (1993), 851-874.

[8] —, - Banach spaces with small spaces of operators, Math. Ann. 307 (1997), 543568.

[9] J. Lindenstrauss and L. Tzafriri, Classical Banach Spaces I. Function Spaces, Springer, New York, 1979.

[10] I. Singer, Bases in Banach Spaces II, Springer, New York, 1981.

Department of Mathematics

IME, University of São Paulo

São Paulo 05315-970 Brazil

E-mail: eloi@ime.usp.br

Received October 14, 2002

Revised version January 2, 2004 